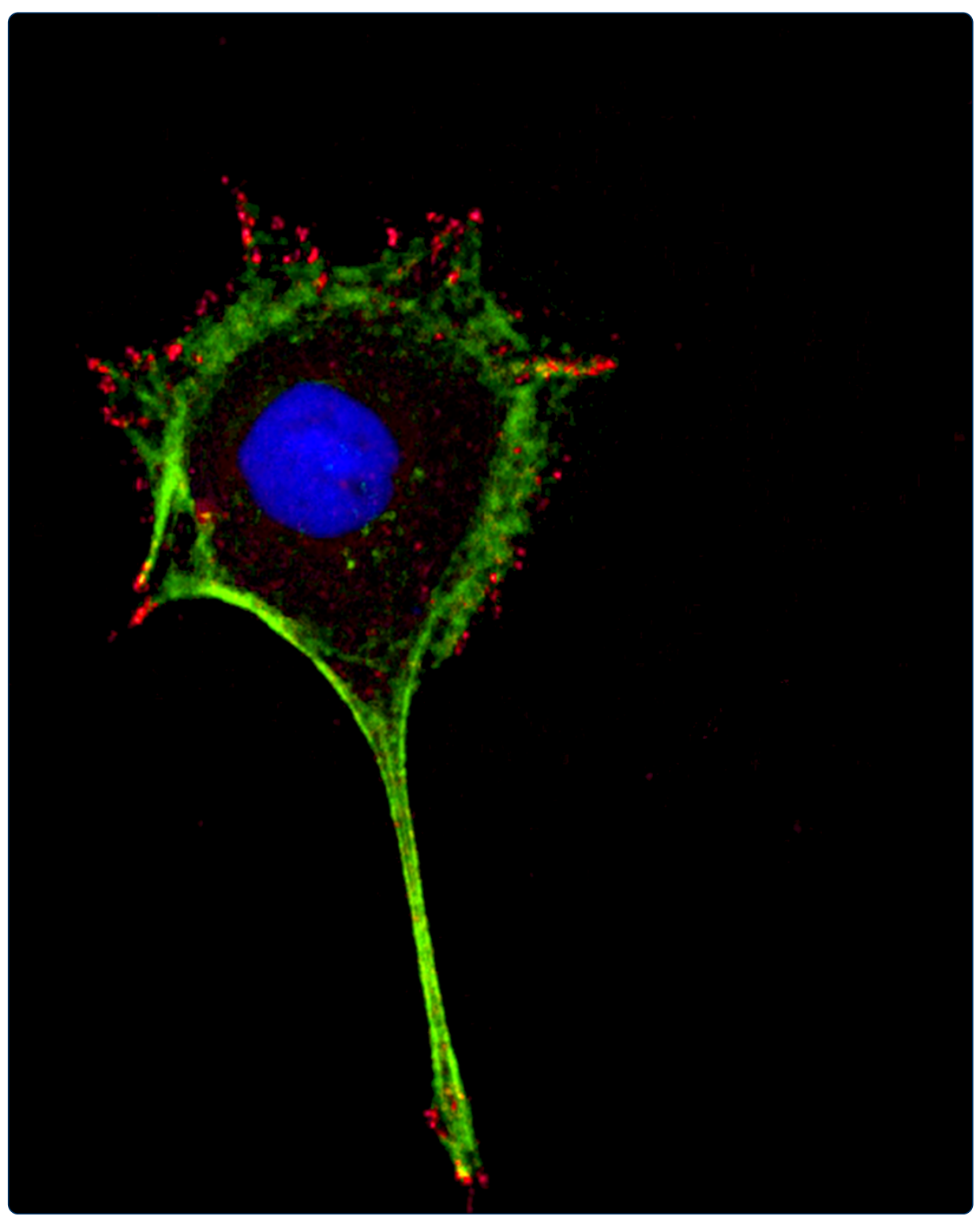

Expression and Y435-phosphorylation of Abelson interactor 1 (Abi1) promotes tumour cell adhesion, extracellular matrix degradation and invasion by colorectal carcinoma cells

Steinestel et al. 


\title{
Expression and Y435-phosphorylation of Abelson interactor 1 (Abi1) promotes tumour cell adhesion, extracellular matrix degradation and invasion by colorectal carcinoma cells
}

Konrad Steinestel ${ }^{1,2^{*}}$, Silke Brüderlein ${ }^{2}$, Jochen K Lennerz ${ }^{2}$, Julie Steinestel ${ }^{3}$, Klaus Kraft ${ }^{4}$, Christian Pröpper ${ }^{5}$, Viktor Meineke ${ }^{1}$ and Peter Möller ${ }^{2}$

\begin{abstract}
Background: The Abelson tyrosine kinase (c-Abl) inhibitor STI571 (Glivec ${ }^{\oplus}$ ) has been shown to effectively inhibit colorectal cancer cell migration and invasion. The c-Abl substrate abelson interactor 1 (Abi1) is a key regulator of actin reorganization and upregulated in colorectal carcinoma. The specific role of Abi1 in relation to extracellular matrix degradation and effects of targeting Abi1 phosphorylation have not yet been examined. Here, we investigated the role of Abi1 in relation to invasive properties in colorectal cancer.

Methods and results: In 56 primary human colorectal carcinoma samples, we found overexpression of Abi 1 in $39 \%$ at the invasive edge of the tumour, associated with an infiltrative phenotype and high-grade tumour cell budding $(p=0.001)$. To explore the role of Abi1 in vitro, we employed the Abi1 expressing and KRAS-mutated CHD1 model and performed matrix degradation assays that showed Abi1 localization at specific sites of matrix degradation. Moreover, quantification of matrix dissolution demonstrated suppression after RNAi knockdown of Abi1 by 95\% ( $p=0.001$ ). Importantly, treatment with STI571 did abolish Abi1 Y435-phosphorylation, suppressed the matrix dissolution, decreased fibronectin attachment, and suppressed cell invasion through reconstituted extracellular matrix.
\end{abstract}

Conclusion: Our data indicate that phosphorylated Abi1 contributes to the invasive properties of colorectal cancer.

Keywords: Abelson interactor 1 (Abi1), Colorectal carcinoma, Extracellular matrix (ECM), Invasion, Metastasis

\section{Background}

Colorectal carcinoma (CRC) is the second leading cause of death from cancer in the western world in both women and men [1]. Patient prognosis is in most cases determined by the ability of tumour cells to invade into surrounding tissue, gain access to blood vessels and form distant metastases after dissemination with the blood stream. Accordingly, a dissociative tumour growth pattern with single-cell infiltration and high-grade tumour cell

\footnotetext{
* Correspondence: konrad@steinestel.com

'Bundeswehr Institute of Radiobiology, Neuherbergstr. 11, 80937 Munich, Germany

${ }^{2}$ Institute of Pathology, University Hospital, Ulm, Germany

Full list of author information is available at the end of the article
}

budding has been shown to be associated with poor prognosis in colorectal cancer [2,3].

Tumour cells facilitate extracellular matrix (ECM) degradation and invasion through the formation of specialized cytoplasmic protrusions, so-called invadopodia [4]. These consist of parallel actin bundles on the basis of a branched actin meshwork and are enriched in proteins that are involved in actin remodelling, including Cortactin, formins of the Diaphanous family and class I nucleation-promoting factors (NPFs) such as WASP or $\mathrm{N}-$ WASP [5]. Actin reorganization is tightly regulated; actin-related proteins (such as Arp2/3) that facilitate actin branching underlie the control of signalling cascades modulating the activity of NPFs and small GTPases [6]. 
Besides providing the structural basis for invadopodia, branched actin filaments give rise to broad-based lamellipodia that are a prerequisite for cellular migration $[5,6]$.

Abelson interactor 1 (Abi1) is a $65 \mathrm{kD}$-substrate of the eponymous Abelson non-receptor tyrosine kinase (c-Abl). The protein is almost ubiquitously expressed with a main focus in the central nervous system [7]. It has been shown that Abi1 plays a central role in synaptic maturation, since knockdown experiments in cultured rat hippocampal neurons led to an immature, filopodia-like synaptic phenotype [8]. In a previous study, we could show that heterogeneous nuclear ribonucleoprotein $\mathrm{K}$, a $65 \mathrm{kD}$ protein that has the ability to act as a translational regulator via binding to mRNA transcripts, interacts with Abil in hippocampal neurons and exerts a similar effect on synaptic maturation [9]. The role of Abi1 in cytoskeletal dynamics during lamellipodia formation has been linked to its ability to join the WAVE multiprotein complex including Abi1, Nap1, HSPC300 and Sra/PIR121 [10,11]; the active complex brings bound Arp $2 / 3$ and actin monomers into close proximity, thus enhancing actin polymerization [12]. Accordingly, Abi1-deficient mouse embryos show malformations in the developing heart and brain and die around embryonic day 11; furthermore, they show decreased integrity and function of the WAVE complex [13]. Interestingly, Abi1 has also been shown to interact with the PI3Kinase, enhancing cytoskeletal reorganization processes in support of cellular migration via activation of Rac activity $[14,15]$. In invading breast cancer cells, Abi1 is required for invadopodia formation and matrix-metalloproteinase 9 secretion [16]. Some effects of Abi1 on cytoskeletal reorganization are dependent on phosphorylation of the protein, and interestingly, application of the Abelson tyrosine kinase inhibitor STI571 (Glivec ${ }^{\circledR}$ ) has been previously found to effectively suppress colorectal cancer cell invasion as well as metalloproteinase-dependent matrix degradation by breast cancer cells [17-19]. Significant antiinvasive effects of STI571 on colon cancer cells have been observed upon application of subtoxic concentrations of the drug in several reports, while the underlying mechanisms remain elusive $[18,20,21]$. In a previous study, we could show that Abi1 is strongly expressed in colorectal adenomas and carcinomas compared to healthy mucosa, and KRAS-mutated hyperplastic polyps show overexpression of the protein compared to their wild-type or $B R A F$ mutated counterparts [22]. Accordingly, overexpression of interaction partners of Abi1 (hnRNP K, N-WASP and WAVE2) has also been previously reported in colorectal carcinoma and CRC metastasis [23-26]. Given the role of Abi1 in actin dynamics, the goal of this study was to investigate its function in extracellular matrix degradation and invasion by CRC cells. Furthermore, we examined the effect of STI571 on Abi1 phosphorylation, metalloproteinase secretion, ECM degradation and colorectal cancer cell invasion.

\section{Results}

Abi1 mRNA expression patterns in gastrointestinal adenocarcinomas

Quantitative Abi1 gene expression data for oesophageal $(\mathrm{n}=13)$, gastric $(\mathrm{n}=21)$, small intestinal $(\mathrm{n}=6)$ and colorectal $(n=505)$ adenocarcinomas obtained from the GeneSapiens transcriptomics database showed comparable expression levels between tumour entities (Figure 1A). Median relative expression levels ranged from about 680 (small intestine adenocarcinoma) to 840 (colorectal carcinoma) arbitrary units.

\section{Clinic-pathologic sample characteristics}

56 colorectal carcinoma surgical specimens were included in the study. All UICC stages, tumour localizations and histopathologic differentiations were represented among the sample cohort (Table 1). Lymph vessel infiltration was present in 19 cases (34\%), while blood vessel infiltration was present in 10 tumours (18\%). $36 \%$ of tumours showed an infiltrating growth pattern; 16 tumours (29\%) displayed high-grade tumour cell budding at the leading edge. Statistical analysis (Fisher's exact test) revealed a significant correlation between infiltrating growth pattern and highgrade tumour cell budding $(\mathrm{p}<0.001)$ and confirmed the association between both infiltrating tumour growth and high-grade tumour cell budding and the presence of lymph or blood vessel invasion by the tumour (L1/V1, $\mathrm{p}=0.019$ and $\mathrm{p}=0.011) .7$ tumours $(13 \%)$ showed loss of mismatch repair protein expression that was significantly associated with expanding, but not infiltrating growth pattern $(\mathrm{p}=0.042)$. Activating $K R A S$ or $B R A F$ mutations were present in $42 \%$ and $4 \%$ of samples, respectively.

\section{Expression of Abi1 at the invasion front of colorectal cancer}

Immunohistochemistry for Abi1 showed strong expression of the protein at the invasive margin of infiltrating, but not expanding CRC (Figure 1B I-III); statistical analysis revealed significant higher Abil staining score at the leading edge of the tumours compared to tumour centre (Figure 1C I; p < 0.001). There was significant overexpression of the protein in tumours displaying an infiltrating growth pattern and high-grade tumour cell budding compared to expanding tumours with a "pushing border" configuration (Figure $1 \mathrm{~B}$ and $1 \mathrm{C} \mathrm{II,} \mathrm{p} \mathrm{<0.001).}$ Abi1 expression correlated with lymph or blood vessel invasion by the tumour (L1V1 status, Figure 1C III; $\mathrm{p}=0.027$ ). Immunofluorescence staining and quantification of staining intensities with an antibody against a phosphorylated isoform of Abi1 (pY435) showed strong nuclear and cytoplasmic positivity in dissociated tumor cells at the invasion front, but only weak staining signals 
A

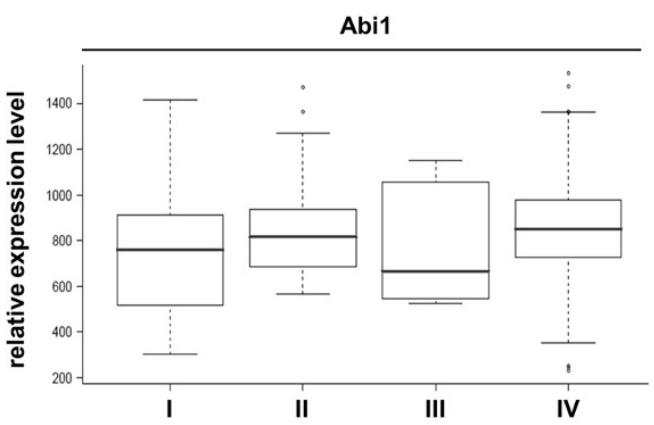

1: esophageal $(n=13)$

2: gastric $(n=21)$

3: small intestine $(n=6)$

4: colorectal $(n=505)$

adenocarcinoma

B

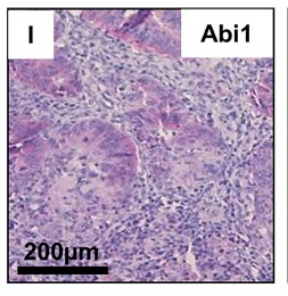

expanding

C 1

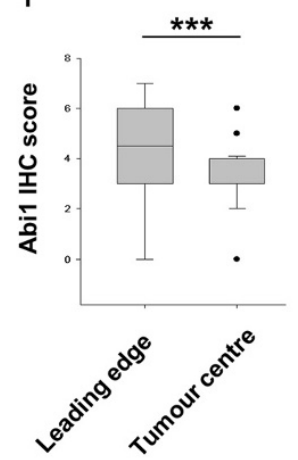

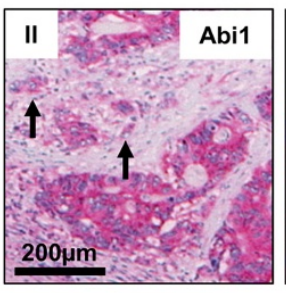

infiltrating

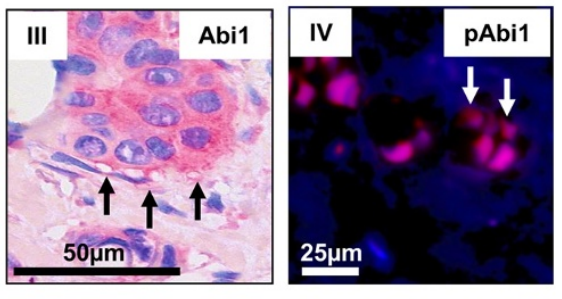

Leading edge
II

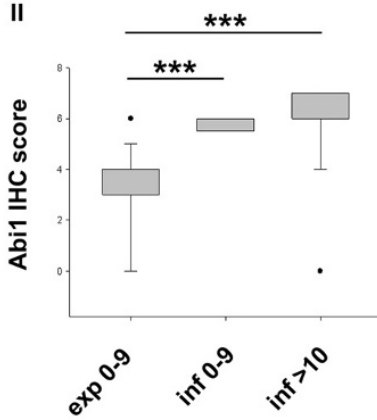

III

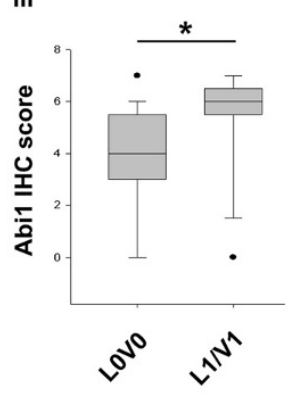

IV

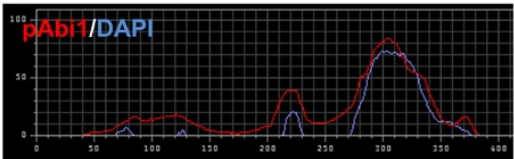

Leading edge

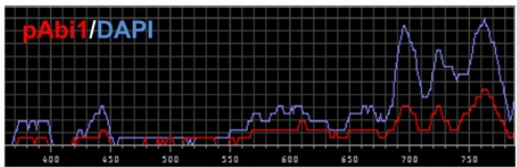

Tumour centre

Figure 1 Abi1 expression analyses. A, in silico gene expression analysis using the GeneSapiens database [31] shows no significant differences in Abi1 gene expression among adenocarcinomas of gastrointestinal origin. B, representative microphotographs of Abi1 immunohistochemistry at the invasive margin of expanding (I) and infiltrating (II) CRC. Tumour cell budding in (II) is indicated by arrows. Greater magnification (III) shows strong Abi1 expression in cytoplasm and podia-like cellular protrusions (arrows). Immunofluorescence staining for phosphorylated Abi1 (p435) shows strong positivity in dissociating tumour cells at the invasive margin (IV). C, statistical evaluation of Abi1 IHC staining scores shows significant higher expression scores at the leading edge compared to tumour centre (I) as well as in tumours with infiltrating growth and high-grade tumour cell budding compared to tumours with expanding growth and low-grade budding (II). Moreover, Abi1 expression score is significantly associated with lymph and/or blood vessel infiltration by the tumour (III). Quantification of staining intensities (IV) shows stronger cytoplasmic as well as nuclear positivity for phosphorylated Abi1 (pY435) at the invasive margin compared to the tumour centre. IHC, immunohistochemistry; exp, expanding growth; inf, infiltrating growth; 0-9, 10: intensity of tumour cell budding in an x250 visual field. Scale bars as indicated. ${ }^{*} p<0.05$; ${ }^{* *} p<0.001$. 
Table 1 Clinic-pathologic sample characteristics

\begin{tabular}{|c|c|}
\hline No. of patients & 56 \\
\hline No. of samples & $56(100 \%)$ \\
\hline Median age (years) & $72 \pm 10.9$ \\
\hline \multicolumn{2}{|l|}{ Gender } \\
\hline Male & $33(59 \%)$ \\
\hline Female & $23(41 \%)$ \\
\hline \multicolumn{2}{|l|}{ Location $(n=55)$} \\
\hline Right & $16(29 \%)$ \\
\hline Left & $39(71 \%)$ \\
\hline \multicolumn{2}{|l|}{ Grading } \\
\hline Well & $6(11 \%)$ \\
\hline Moderate & $24(43 \%)$ \\
\hline Poor & $26(46 \%)$ \\
\hline \multicolumn{2}{|l|}{ Growth pattern } \\
\hline Expanding & $36(64 \%)$ \\
\hline Infiltrating & $20(36 \%)$ \\
\hline \multicolumn{2}{|l|}{ Budding intensity } \\
\hline Low-grade & $40(71 \%)$ \\
\hline High-grade & $16(29 \%)$ \\
\hline \multicolumn{2}{|l|}{ Lymph vessel infiltration } \\
\hline LO & $37(66 \%)$ \\
\hline L1 & 19 (34\%) \\
\hline \multicolumn{2}{|l|}{ Blood vessel infiltration } \\
\hline vo & $46(82 \%)$ \\
\hline V1 & $10(18 \%)$ \\
\hline \multicolumn{2}{|l|}{ MMR protein expression } \\
\hline MMR proteins expressed & $49(87 \%)$ \\
\hline Loss of MLH1 + PMS2 & $6(11 \%)$ \\
\hline Loss of PMS2 & $1(2 \%)$ \\
\hline \multicolumn{2}{|l|}{ KRAS status $(\mathrm{n}=55)$} \\
\hline KRAS wild-type & $32(58 \%)$ \\
\hline KRAS G12D & $9(16 \%)$ \\
\hline KRAS G12V & $7(13 \%)$ \\
\hline KRAS G12C & $1(2 \%)$ \\
\hline KRAS G12S & $1(2 \%)$ \\
\hline KRAS G13D & $5(9 \%)$ \\
\hline \multicolumn{2}{|l|}{ BRAF status } \\
\hline$B R A F$ wild-type & $54(96 \%)$ \\
\hline BRAF V600E & $2(4 \%)$ \\
\hline \multicolumn{2}{|l|}{ UICC Stage $(n=55)$} \\
\hline I & $16(29 \%)$ \\
\hline II & $15(27 \%)$ \\
\hline III & 17 (31\%) \\
\hline IV & $7(13 \%)$ \\
\hline
\end{tabular}

in the tumour body (representative images, Figure 1B IV and Figure $1 \mathrm{C}$ IV).

\section{Expression and phosphorylation of Abi1 in CHD1 cells}

CHD1 colorectal carcinoma cells are positive for Abi1, hnRNP $\mathrm{K}$ and Laminin $5 \gamma^{2}$ in Western immunoblotting (Figure 2A and Additional file 1: Figure S1A). The antibody against Laminin 5 detected two bands migrating at $100 \mathrm{kD}\left(\mathrm{L} 5 \mathrm{\gamma}^{\prime}\right.$ ) and $85 \mathrm{kD}$ (L5 $\left.\gamma 2 \mathrm{x}\right)$, indicating cleavage of the protein [27]; E-cadherin was not expressed at a detectable level in CHD1 whole cell lysate. These findings could be confirmed in IF microscopy (Additional file 1: Figure S1B). Further immunofluorescence analyses showed localization of Cortactin and Abil to the outer rim of lamellipodia-like cellular protrusions (Figure 2B I and II). Immunofluorescence staining with an antibody against Y435-phosphorylated Abil showed strand-like positivity along the growth axis of cellular protrusions (Figure 2B III); treatment with $10 \mu \mathrm{M}$ of the Abl tyrosine kinase inhibitor STI571 markedly reduced Abi1 and pAbi1 positivity in peripheral cellular compartments with remaining central (perinuclear) positivity for Abi1.

\section{Fibronectin cell adhesion assay}

When seeded onto fibronectin-coated coverslips, CHD1 cells showed outgrowth of broad-based lamellipodia with peripheral, strand-like positivity for phosphorylated Abi1 (Figure 2C I and II). $10 \mu \mathrm{M}$ STI571 significantly impaired lamellipodia formation and cellular adhesion on fibronectin (Figure $2 \mathrm{C}$ III-V, $\mathrm{p}<0.001$ ).

\section{Gelatine-based ECM degradation assay}

When CHD1-cells were seeded out on fluorescent gelatinecoated coverslips, Cortactin and Abil localized to peripheral foci of matrix degradation (Figure 3A I and II). This was confirmed in $3 \mathrm{D}$ surface reconstruction, where rimlike Abi1 positivity in the cell periphery co-localized with sites of matrix cleavage (Figure 3A III). Application of $10 \mu \mathrm{M}$ STI571 led to a complete arrest in matrix degradation (Figure 3A IV).

\section{Effect of tyrosine kinase inhibition and Abi1 RNAi} knockdown on ECM degradation

To further investigate the role of (phosphorylated) Abi1 in ECM degradation, CHD1 cells were seeded out on fluorescent gelatine-coated coverslips and either treated with $10 \mu \mathrm{M}$ STI571/DMSO or transfected with RNAi targeting Abi1/vector control; after $24 \mathrm{~h}$, degradation area was measured and degradation coefficients were calculated (see "Materials and methods" section for details). Statistical analysis revealed that $10 \mu \mathrm{M}$ STI571 as well as Abi1 RNAi knockdown significantly reduced the ability of CHD1 cells to lyse extracellular matrix compared 


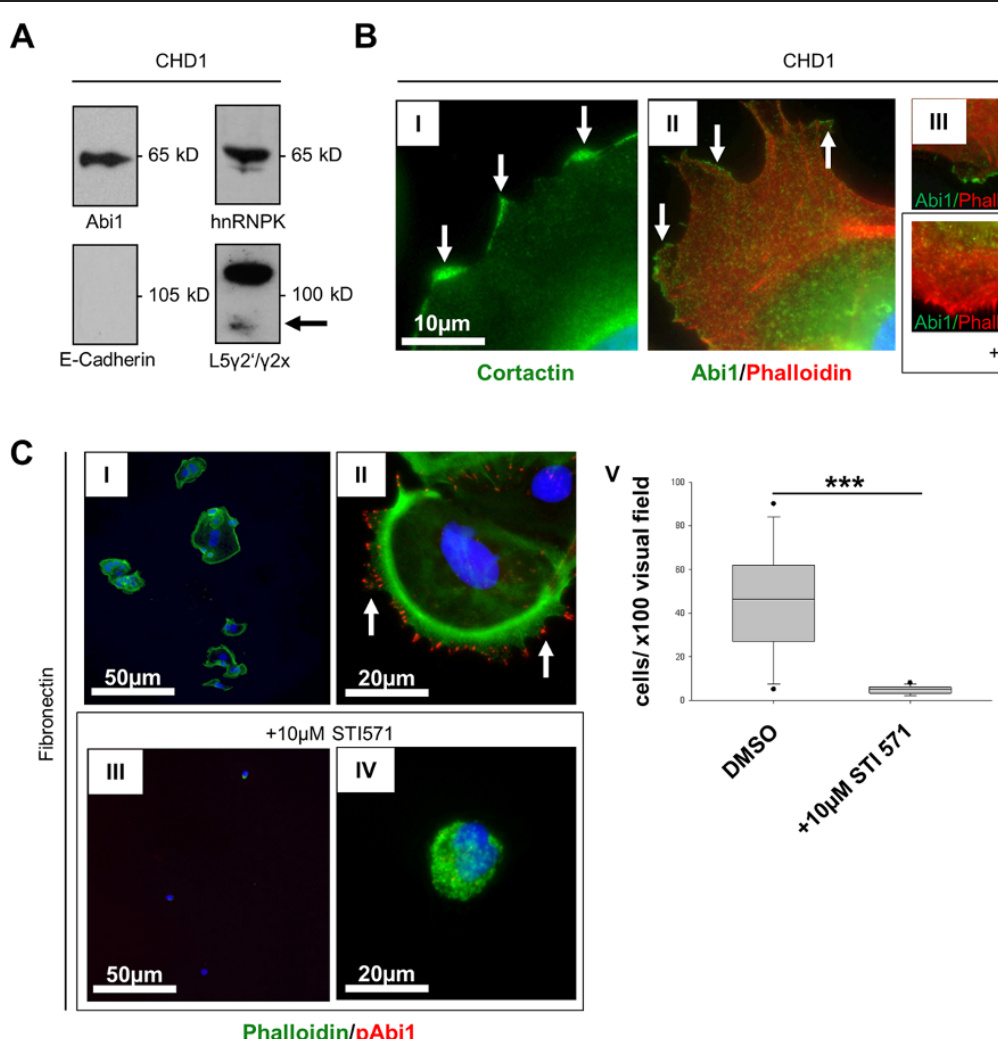

Figure 2 Abi1 expression and subcellular localization in CHD1 colorectal carcinoma cells. A, western immunoblotting of CHD1 whole cell lysate shows expression of Abi1 and hnRNP K as well as a 100/85 kD double-band for Laminin5, but no detectable levels of E-Cadherin. B, IF microscopy shows peripheral, rim-like distribution of Cortactin (I) and Abi1 (II). Signals for both Abi1 and Y435-phosphorylated Abi1 in the cell periphery, but not in the perinuclear region, are extinct after treatment with $10 \mu \mathrm{M}$ STI571 (III). C, fibronectin adhesion assay shows cellular attachment with lamellipodial protrusions (I) and peripheral positivity for Y435-phosphorylated Abi1 (II) under control conditions, but only few attached cells (III) with condensed actin and negativity for pAbi1 after treatment with $10 \mu \mathrm{M}$ STI571 (IV). Cell attachment was significantly reduced after treatment with STI571 (M). DMSO, dimethyl sulfoxide. Scale bars as indicated. ${ }^{* * *} p<0.001$.

to DMSO-treated or vector control-transfected cells (Figure 3B and C; $\mathrm{p}<0.001$ ).

\section{Effect of tyrosine kinase inhibition on Abi1 phosphorylation, matrix metalloproteinase 9 secretion and tumour cell invasion}

To confirm inhibition of Abi1 Y435-phosphorylation as well as specificity of STI571, we performed western immunoblotting after treatment with DMSO control or STI571. The band for phosphorylated Abi1 that was observed under control conditions was extinct after treatment with 5 and $10 \mu \mathrm{M}$ STI571, while total Abi1 was strongly expressed (Figure 4A and Additional file 1: Figure S3A). There was no decrease, but a slight increase in Erk1/2 expression and phosphorylation, while protein levels of cleaved caspase-3 (CC-3) were barely detectable upon treatment with 5 and $10 \mu \mathrm{M}$ STI571 (Figure 4A); application of $50 \mu \mathrm{M}$ STI571 led to a strong increase in CC-3 expression (Additional file 1: Figure S3B). Beta-actin was used as a loading control.
Changes in matrix metalloproteinase 9 (MMP-9) secretion were assessed using ELISA (Figure 4B) in cell culture supernatant from CHD1 cells that had been treated with STI571 or DMSO control as indicated. There was a slight decrease in MMP-9 secretion that was not statistically significant $(\mathrm{p}=0.162)$.

The ability of CHD1 cells to invade through a reconstituted basement membrane under increasing concentrations of STI571 was investigated applying the ECMatrix Cell Invasion Assay (EMD Millipore, Darmstadt, GER). Invaded cells were spun on a glass slide, counterstained with DAPI and counted automatically. $10 \mu \mathrm{M}$ of STI571 reduced the invasive potential of CHD1 cells up to $80 \%$ (Figure 4C; $\mathrm{p}<0.05$ ).

\section{Discussion}

Abelson interactor 1 (Abi1), a $65 \mathrm{kD}$ substrate of the eponymous Abelson tyrosine kinase (c-Abl), has been initially described as a regulator of actin dynamics and synaptic maturation in neurons $[7,8,28]$. In recent studies, 

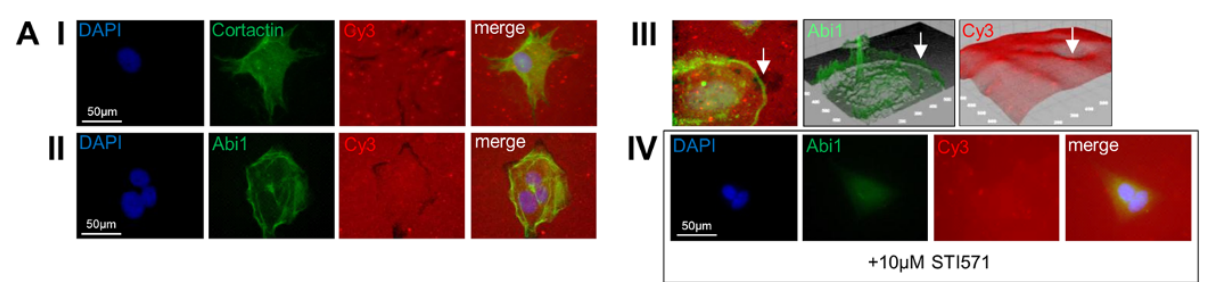

B

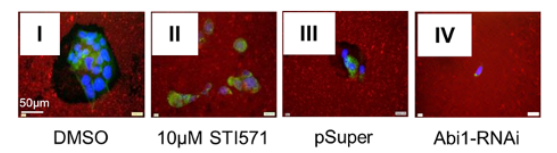

C

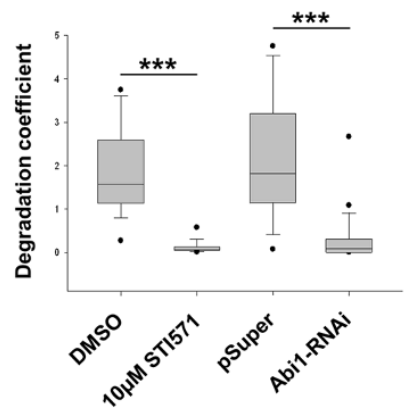

Figure 3 Gelatine-based ECM degradation assay. A, CHD1 cells seeded on Cy3-conjugated gelatine matrix show cytoplasmic and peripheral positivity for Cortactin (I) and Abi1 (II) at the sites of matrix degradation. This is confirmed in 3D surface reconstruction for Abi1 (III, arrow). Application of 10 MM STI 571 leads to loss of Abi1 staining in cell periphery together with lack of gelatine degradation (IV). B and C, Application of STI571 in a concentration of 10 MM (I: DMSO control, II: STI571) as well as Abi1 RNAi knockdown (III: pSuper vector control, IV: Abi1 RNAi) inhibits ECM degradation as shown by a significant reduction in degradation coefficient. DMSO, dimethyl sulfoxide. Scale bars as indicated. ${ }^{* *} p<0.001$.

A

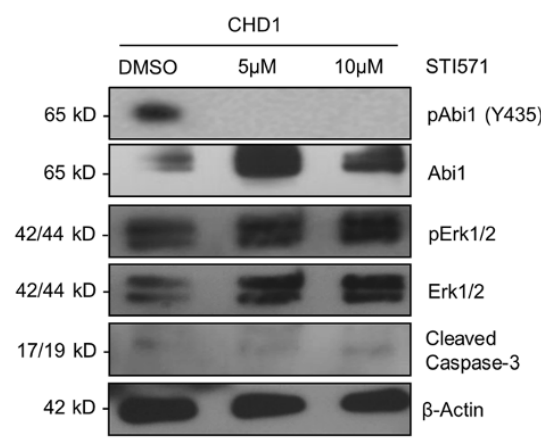

C

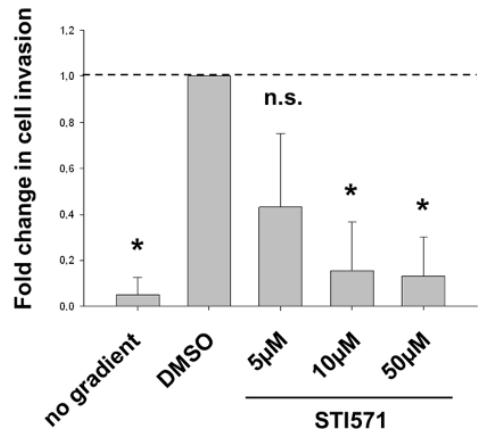

B

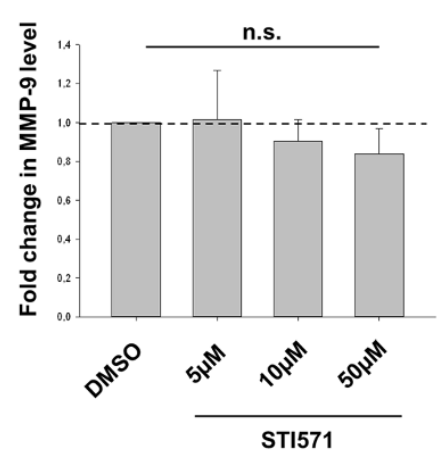

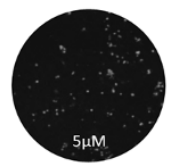

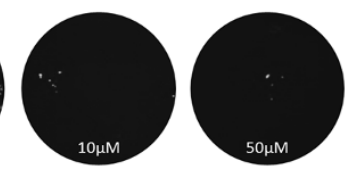

STI571

Figure 4 Functional role of Abi1 phosphorylation in MMP-9 secretion and CRC cell invasion. A, western immunoblotting of CHD1 cell lysate after control (DMSO) treatment or application of ST1571 as indicated confirms inhibition of Abi1 Y435-phosphorylation through STI571, while levels of total Abi1, phospho-Erk1/2 and total Erk1/2 slightly increase upon STI treatment. Levels of cleaved caspase-3 are barely detectable. B, MMP-9 ELISA from cell culture supernatant after control (DMSO) treatment or application of ST1571 shows a non-significant decrease in MMP-9 secretion after treatment with 5-50 $\mu \mathrm{M}$ STI571. C, increasing concentrations of ST1571 inhibit CHD1 CRC cell invasion through reconstituted basement membrane in a dose-dependent manner. Representative microphotographs (right) show DAPI-counterstained cells that invaded through the sealed pores and were harvested from the lower chamber. DMSO, dimethyl sulfoxide; MMP-9, matrix metalloproteinase 9; n.S., not significant. ${ }^{*} p<0.05$. 
overexpression of the protein has been linked to increased metastatic potential in breast and ovarian cancer, and several studies described an inhibitory effect of low concentrations of the c-Abl inhibitor STI571 on colorectal carcinoma cell invasion $[18,20,21,29,30]$. Our own group found overexpression of Abi1 in KRAS-mutated colonic precursor lesions and during colon carcinogenesis [22]; therefore, the aim of this study was to evaluate the expression and functional role of Abi1 in colorectal cancer (CRC) invasion as a prerequisite to tumour cell invasion and metastasis.

Gene expression levels were assessed using data from 545 tissue samples from the GeneSapiens in silico database [31] and showed no significant differences in Abi1 gene expression among adenocarcinomas of gastrointestinal origin. This finding is consistent with protein expression data obtained from the human protein atlas [32], another in silico database for tissue microarraybased protein expression patterns $[33,34]$. In that database, $86 \%$ of gastric and colorectal tumour specimens showed moderate to strong Abil staining intensity with the identical antibody that was used in the present study. Taken together, these large-scale expression analyses confirm the strong expression of Abi1 that we previously reported for CRC among diverse adenocarcinomas of the gastrointestinal tract [22]. However, Abi1 mRNA as well as protein expression data reveals great intra- and intertumoural heterogeneity. Therefore, we analysed Abi1 expression at the leading edge and in the tumour centre of 56 invasive CRCs and found that expression of the protein correlated significantly with infiltrating growth pattern and high-grade tumour cell budding, both characteristics being widely accepted to be associated with aggressive behaviour and poor prognosis in CRC [2,3]. We could confirm the correlation between infiltrative growth and high-grade tumour cell budding as well as lymph or blood vessel invasion by the tumour in our sample set, supporting the assumption that these morphologic features herald an aggressive tumour phenotype. Lymphatic and blood vessel invasion, representing significant prognostic variables in CRC, were independently associated with strong expression of Abi1 at the invasive margin of the tumours [35]. These findings are consistent with results obtained from other tumour entities, since it has been shown that overexpression of Abi1 is associated with early recurrence and worse survival in breast cancer; in ovarian cancer, Abi1 is an essential factor in a protein tricomplex indispensable for metastatic capability of tumour cells [29,30]. Moreover, immunofluorescence microscopy revealed a strong staining signal for a phosphorylated isoform of Abi1 (Y435) at the leading edge of infiltrating tumours with high expression of Abi1, indicating a role for Abi1 tyrosine phosphorylation in CRC cell invasion. To further investigate the functional role of Abi1 in CRC, we analysed expression and subcellular localization of the protein in CHD1 cells carrying an activating KRAS G13D mutation. Initially, the cell line had been selected because of its high Abil expression level [22], but in the present study, additional immunoblotting experiments showed cleavage of Laminin $5 \gamma 2$ and loss of E-cadherin expression in CHD1 cells. Both features are consistent with a promigratory, epithelial-mesenchymal-transition-like cellular phenotype that might be linked to constitutively active Ras signalling [36,37]. Accordingly, HDC9 KRAS/BRAF wild-type colorectal carcinoma cells - that weakly express Abi1 [22] - display high levels of E-cadherin and no cleavage of Laminin 5 indicated by a single y2' band migrating at 100-105 kD (Additional file 1: Figure S1A).

Immunofluorescence microscopy showed localization of Abi1 to a peripheral rim around lamellipodia-like cellular protrusions in cultured CHD1 cells, a distribution pattern comparable to the established invadopodia marker Cortactin [4]. The phosphorylated isoform of Abi1 (Abi1-pY435) was detected in strand-like alignments along broad-based cellular protrusions, and both peripheral staining signals were extinct after treatment with $10 \mu \mathrm{M}$ of the Abl kinase inhibitor STI571 (Glivec ${ }^{\circ}$ ). Furthermore, this treatment prevented CHD1 cells from firmly attaching to fibronectin-covered surfaces. To verify the results from IF microscopy, we performed additional immunoblotting experiments and could confirm that the band for Y435-phosphorylated Abi1 was extinct after treatment with STI571, while levels of total Abi1 as well as total and phosphorylated Erk1/2 were even slightly elevated. These findings support the results from IF staining, where Abi1 remained strongly expressed upon STI571 treatment, and point towards a cellular redistribution of Abi1 rather than a decrease in protein expression upon kinase inhibition. Furthermore, as shown by Erk1/2 phosphorylation, signalling activity along the central MAPK pathway was not inhibited, but slightly enhanced by STI571 in the given concentrations. This is consistent with our previous results showing Abi1 upregulation upon Ras-MAPK signalling in colorectal precursor lesions, and might point towards an alternative signalling route via MAPK upon application of STI [22]. Western immunoblotting for cleaved caspase- 3 gave no evidence for the induction of apoptosis upon application of 5 or $10 \mu \mathrm{M}$ STI571, while there was strong immunoreactivity upon $50 \mu \mathrm{M}$ of the drug, consistent with literature data [21]. It has to be recognised, however, while our results do show a decrease in Abi1 Y435-phosphorylation upon STI571 especially in the cell periphery that might be due to $\mathrm{c}$-Abl inhibition, we did not prove that this tyrosine residue is in fact phosphorylated by c-Abl. This question should be addressed in further experiments applying knockout of $\mathrm{Abl}$ and/or site-directed mutagenesis. 
Invadopodia show a branched actin network closely related to the architecture observed in lamellipodia, but they are able to degrade ECM through secretion of lytic proteinases [4]. When CHD1 cells were seeded upon a fluorescent gelatine matrix, Abi1 and Cortactin localized to peripheral foci of matrix degradation, and application of STI571 as well as RNAi knockdown of Abi1 eliminated the ability of CHD1 cells to lyse the ECM as shown by a significant decrease in degradation coefficients. This is consistent with the described role of Abi1 in breast cancer cell invadopodia formation and might explain the inhibition of colorectal cancer cell migration and invasion through STI571 that has been previously shown $[16,20]$. Therefore, a model where Abi1 and Cortactin act cooperatively in invadopodia at the leading edge of colorectal carcinoma is quite conceivable; phosphorylated Abi1 would then support local actin reorganization via activation of nucleation-promoting factors, giving rise to a branched actin network required for ECM attachment and invadopodia formation (schematically depicted in Figure 5) $[4,16]$. This model is supported by the fact that upregulation of Abi1-interacting formins and class I NPFs has been reported to be associated with enhanced metastasis in CRC, and increased MMP9 secretion upon Abi1 overexpression has been shown in breast cancer $[16,25,38]$. However, in the present study, we could not show a significant decrease in MMP-9 secretion after STI571 treatment, why we conclude that the impact of Abi1 phosphorylation seems to lie in invadopodia formation rather than MMP-9 secretion by CRC cells. It might be discussed if other proteases play a central role during tumour cell invasion in CRC. One hint might be the observed cleavage of Laminin5, since this process is in most part mediated by MMP-1 and MMP-7, and only in little part by MMP-9, in colon cancer [36,39]. In our model, an increase in the local concentration of such lytic enzymes would lead to ECM dissolution and cleavage of Laminin5 (schematically depicted in Figure 5); accordingly, associations between high MMP-1 expression levels and both presence of liver metastases and poor prognosis have been reported in CRC $[40,41]$.

Finally, we show that tumour cell invasion through a reconstituted basement membrane was diminished up to $80 \%$ after application of $10 \mu \mathrm{M}$ STI571. These results are in line with findings from other authors, who described a remarkable decrease in cell migration capacity in response to STI treatment in colon cancer, and indicate that the antiinvasive function of STI571 might at least in part be mediated by inhibition of Abi1 phosphorylation that impairs invadopodia formation by CRC cells $[18,20,21]$.

\section{Conclusions}

Our results show for the first time that Abi1 is overexpressed at the invasive edge of colorectal carcinomas

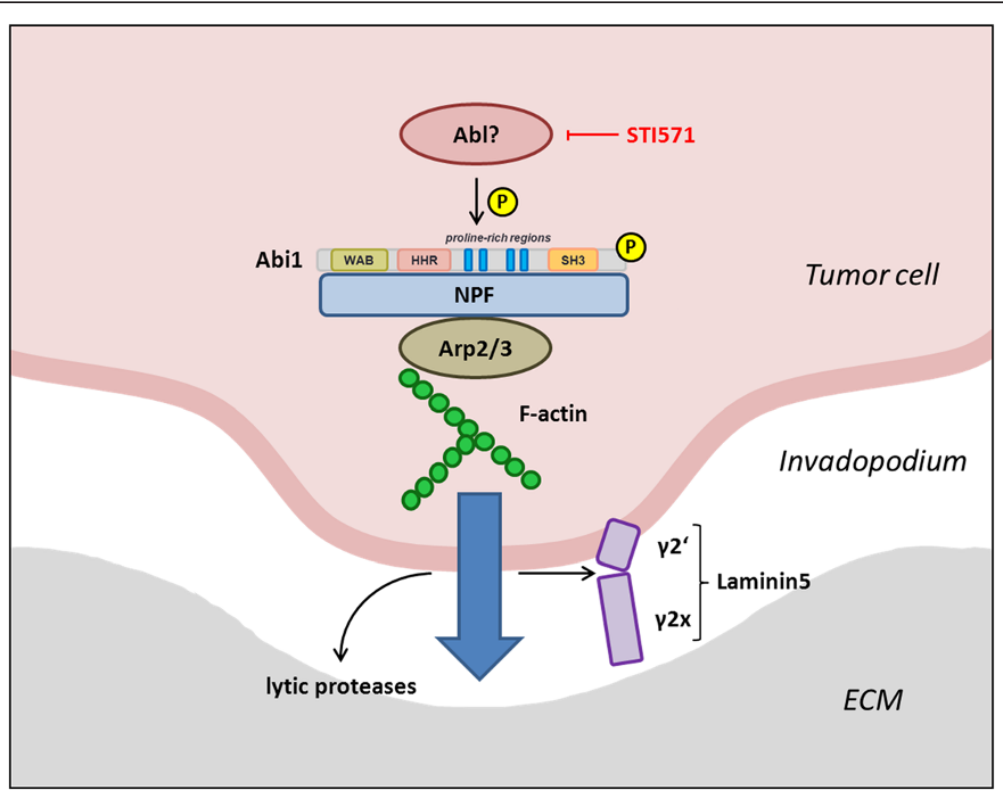

Figure 5 A possible pathway for the functional role of Abi1 phosphorylation in colorectal carcinoma invasion. Y435-Phosphorylation of Abi1 through Abl or another kinase that is targeted by STI571 enhances activity of class I and/or class II NPFs, thus facilitating actin branching and invadopodia formation. Secretion of lytic proteinases at the site of invadopodia formation leads to ECM degradation and cleavage of Laminin5, which supports local tumour cell invasion. Abl, Abelson tyrosine kinase; Abi1, Abelson interactor 1; WAB, Wave-binding domain; HHR, homeobox homology region; SH3, src homology domain 3; NPF, nucleation-promoting factor; Arp2/3, actin-related protein complex; F-actin, filamentous actin; ECM, extracellular matrix. 
that display an aggressive phenotype; furthermore, phosphorylated Abi1 as well as Cortactin localize to foci of matrix degradation in the tumour cell periphery, while treatment with STI571 leads to a depletion of Y435phosphorylated Abi1 in this compartment and suppresses tumour cell attachment to fibronectin as well as ECM degradation and tumour cell invasion in vitro. Finally, our findings support previously published data indicating that STI571 might be a pharmacological option to prevent the gain of an invasive phenotype in colorectal cancer.

\section{Materials and methods GeneSapiens database}

To evaluate Abi1 gene expression patterns among different adenocarcinomas of the gastrointestinal tract, we obtained publicly available expression data for oesophageal $(n=13)$, gastric $(n=21)$, small intestine $(n=6)$ and colorectal $(n=505)$ adenocarcinomas from the GeneSapiens database $[31,42]$. This database contains data from 5 Affymetrix array generations (about 10000 samples, average of 11500 genes per sample) subdivided into healthy, malignant, and non-tumour disease tissue.

\section{Patient samples, clinical data and ethics statement}

All patient samples have been submitted to the Institute of Pathology, Bundeswehrkrankenhaus Ulm, for routine diagnostic purposes between 2006 and 2012. Clinic-pathological data including TNM/UICC stages, tumour differentiation, infiltration patterns and presence of blood vessel invasion was obtained from the pathology report archive of the Institute of Pathology, Bundeswehrkrankenhaus Ulm, and are summarized in Table 1. All samples have been anonymised thoroughly for the use in the study. This study has been approved by the local ethics committee of the University of Ulm (No. 162/13).

\section{KRAS/BRAF mutation testing and MMR protein immunohistochemistry}

Mutation status of $K R A S$ (codons 12, 13 and 61) and $B R A F$ (codon 600) was performed using KRAS/BRAF strip assays (Vienna Labs, Vienna, AUT and AID diagnostics, Strassberg, GER) as previously described [22]. Shortly, DNA was extracted from $30 \mathrm{~mm}$-thick slides that were cut off paraffin blocks, xylene treated and ethanol washed. PCR amplification of KRAS and BRAF with biotinylated primers and hybridization-based mutation analysis was done according to the manufacturers' protocols. Immunohistochemistry for mismatch repair (MMR) proteins MLH-1, MSH-2, MLH-6 and PMS-2 was performed on BenchMark Autostainer (Ventana, Tucson, USA) using prediluted mouse monoclonal antibodies (Ventana, Tucson, USA).

\section{Evaluation of invasion patterns and immunohistochemical analysis}

Invasion pattern of each tumour was assessed applying the Jass classification modified by Ueno et al., since this scheme has been shown to represent a good index to estimate the aggressiveness of colorectal adenocarcinoma [2,3]. The classification scheme divides invasive margins into "expanding" (pushing/well circumscribed) or "infiltrating" (diffuse, widespread penetration) and takes into account the grade of tumour "budding". As in the original publication, budding was defined as isolated cells or clusters of less than five cancer cells; the number of budding foci was counted in a field where budding intensity was considered maximal using a $\times 25$ objective lens. 0-9 foci were defined as low-grade, while $\geq 10$ foci were defined as high-grade tumour budding.

Abi1 immunohistochemistry was performed on 1-2 slides that displayed the invasive margin of the tumours using a mouse monoclonal antibody (clone 1B9, MBL, Woburn, USA; conc. 1:200). Staining intensity was evaluated at the invasion front of the tumours using the scoring system described by Carpenter et al. [23]. The intensity of immunostaining was graded as negative $=0$, weak $=1$, moderate $=2$ or strong $=3$. The proportion of positively stained cells was assessed as no cells $=0$, $1-25 \%$ of cells $=1,26-50 \%=2,51-75 \%=3$ and $76-$ $100 \%=4$. The numbers representing intensity and percentage of stained cells were added together and the result will be referred to as score.

\section{Cell culture, pharmacological treatment, MMP-9 ELISA and immunoblotting}

CHD1 cells were obtained from the Institute of Pathology, University of Ulm, and have been previously published $[22,43]$. Cells were kept under sterile conditions in RPMI-1640 medium supplemented with 10\% FCS. STI571 was dissolved in DMSO and applied in the indicated concentrations 24 or $48 \mathrm{~h}$ prior to cell lysis/fixation. Cell culture supernatants were collected and delivered to matrix-metalloproteinase 9 ELISA using the Human MMP-9 Quantikine ELISA Kit (R\&D Systems, Wiesbaden, Germany) while adherent cells were lysed in standard lysis buffer containing protease and phosphatase inhibitors (Cell Signaling Tech., Boston, USA). Immunoblotting was performed according to standard methods using the following antibodies: mouse monoclonal to Abi1 (1:200), MBL, Woburn, USA; mouse monoclonal to hnRNP K (1:500), Sigma-Aldrich, St. Louis, USA; rabbit polyclonal to E-Cadherin (1:250), Sigma-Aldrich, St. Louis, USA; rabbit polyclonal to Laminin $5 \gamma 2$ (1:250), Sigma-Aldrich, St. Louis, USA; rabbit monoclonal to Abi1-pY435 (1:250), Abcam, Cambridge, UK; rabbit monoclonal to p44/42 (Erk1/2, 1:1000), phospho-p44/42 (phospho-Erk1/2, 1:2000) and cleaved caspase-3 (1:1000), all from Cell Signaling Tech., Boston, 
USA; mouse monoclonal to actin (1:1000), Thermo Scientific, Rockford, USA.

\section{Immunofluorescence (IF) staining and fibronectin adhesion assay}

For immunofluorescence (IF) staining's, CHD1 colorectal carcinoma cells were seeded onto 4-well chamber slides (Nunc, Wiesbaden, GER), and staining was carried out as previously described [44]. In short, cells were fixed in ice-cold $4 \%$ paraformaldehyde, permeabilised with $0.2 \%$ Triton-X 100 and incubated with 5\% FCS in PBS. Alternatively, FFPE slides from tumour centre and invasive margin were deparaffinised, permeabilised and blocked with 5\% FCS in PBS. Afterwards, slides were incubated with one or two of the following primary antibodies for $1 \mathrm{~h}$ at room temperature: mouse monoclonal to Abi1 (1:200, MBL, Woburn, USA); rabbit monoclonal to Cortactin (1:250, Sigma-Aldrich, St. Louis, USA); rabbit polyclonal to Abi1-pY435 (1:250, Abcam, Cambridge, UK); mouse monoclonal to hnRNP K (1:250), SigmaAldrich, St. Louis, USA; rabbit polyclonal to E-Cadherin (1:250), Sigma-Aldrich, St. Louis, USA; rabbit polyclonal to Laminin5y2 (1:250), Sigma-Aldrich, St. Louis, USA. After washing, slides were incubated with secondary antibodies (goat polyclonal anti-mouse, Texas Redconjugated, Abcam, Cambridge, UK; goat polyclonal anti-rabbit, DyLight ${ }^{\circ} 488$ - conjugated, Vector Laboratories, Burlingame, USA; goat polyclonal anti-mouse IgM Antibody, Cy2-conjugated, Biozol, Eching, GER; goat polyclonal anti-mouse IgG $(\mathrm{H}+\mathrm{L}), \mathrm{F}(\mathrm{ab}$ ') 2 Fragment, Alexa Fluor ${ }^{\circ}$ 488-conjugate, Life technologies, Darmstadt, GER). For phalloidin staining, FITC/ TexasRed-coupled Phalloidin (Life technologies, Darmstadt, GER) was added after 30 min incubation at RT in the dark. Slides were mounted in Vectashield aqueous mounting medium containing DAPI (Vector Laboratories, Burlingame, USA). For the fibronectin adhesion assay, chamber slides were covered with Human Plasma Fibronectin (Life technologies, Darmstadt, GER, conc. $25 \mu \mathrm{g} / \mathrm{ml}$ ) for $2 \mathrm{~h}$ at $37^{\circ} \mathrm{C}$. Cells were seeded in serum-free medium with either $10 \mu \mathrm{M}$ STI571 or DMSO (control). 6 hours after seeding, slides were 6 times rinsed with PBS, and attached cells underwent fixation and immunofluorescence staining as described above. Image acquisition as well as quantification of IF staining intensities was performed using a Zeiss Axioplan microscope and Isis software (MetaSystems, Altlussheim, GER); for fibronectin adhesion assays, quantification of attached cells in 12 visual fields $(\times 10$ objective lens) was done using Image J image analysis software (National Institutes of Health, Bethesda, USA).

\section{ECM degradation assay}

For visualization of ECM degradation, the $\mathrm{QCM}^{\mathrm{six}}$ Gelatine Invadopodia Assay (EMD Millipore, Darmstadt,
GER) was applied according to the manufacturer's protocol. Briefly, 8-well-chamberslides (Nunc, Wiesbaden, GER) were covered with Poly-L-Lysine, Glutaraldehyde and Cy3-labeled fluorescent gelatine. CHD1 colorectal carcinoma cells were seeded in fresh growth medium at $500 \mu \mathrm{L} /$ well and allowed to attach for $6 \mathrm{~h}$ before RNAi transfection/pharmacological treatment. After another $36 \mathrm{~h}$ (transfection)/24 h (treatment), medium was removed and slides were fixed in $4 \%$ PFA for immunofluorescence staining as described above. Qualitative and quantitative image analysis was done using Image J image analysis software (National Institutes of Health, Bethesda, USA) [45]. 3D surface plots were generated using the 3D surface plot add-in for Image J (Kai Uwe Bartel, HTW Berlin, GER). Quantification of extracellular matrix degradation was done using Image analysis software as previously described [46]. High threshold was set for the DAPI channel and counted as "particles" for total cell count and the Cy2/Alexa488/FITC signal for total cell surface area per field of view. Conversely, low intensity threshold was set for diminished Cy3-gelatin signal to enable quantification of total degradation area. The ratio between degradation area and cell area was defined as the degradation coefficient (DC). For quantification of matrix degradation after RNAi knockdown and pSuper vector control, only the degradation coefficient of cells that showed cytoplasmic pSuper marker fluorescence was calculated. For DMSO- or STI571-treated cells, Phalloidin counterstaining was used to visualize cell surface area.

\section{Small interference RNA (RNAi) experiments}

Knockdown of Abil was achieved by RNAi according to published methods using the pSuper vector (OligoEngine, Seattle, USA). Complementary oligonucleotides were annealed and inserted into the Hind III/Bgl II sites of the pSUPER vector. Initially, the Abil construct had been designed complementary to rattus norvegicus Abi1 mRNA [GenBank NM_024397]. NCBI blast sequence analysis revealed $100 \%$ homology with homo sapiens Abil over a span of 16 nucleotides (sequence in italics), a feature which has been previously reported to be an even more effective trigger of RNA induced silencing complex (RISC) formation than 19-nt-long cores [47]; Abi1 RNAi: 5'-GAT CCC CAG GCT ACA GAC AAG AGG AAT TCA AGA GAT TCC TCT TGT CTG TAG CCT TTT TTG GAA A-3' (sense) and 5'- AGC TTT TCC AAA AAA GGC TAC AGA CAA GAG GAA TCT CTT GAA TTC CTC TTG TCT GTA GCC TGG G-3' (antisense). Protein knockdown efficacy of the construct in human CHD1 cells was tested using immunofluorescence analysis and western immunoblotting (Additional file 1: Figure S2A and B). Transfections were performed using the Optifect transfection reagent (Qiagen, Hilden, GER) according to the manufacturer's protocol in serumfree medium $(4 \mu \mathrm{l}$ per $\mu \mathrm{g}$ DNA). 


\section{Cell invasion assay}

To evaluate ability of colorectal carcinoma cells to invade into ECM, cells were seeded onto the polycarbonate membranes of cell culture inserts with $8 \mu \mathrm{m}$-pores sealed with ECMatrix, a reconstituted basement membrane matrix derived from Engelbreth Holm-Swarm (EHS) mouse tumour (ECMatrix Cell Invasion Assay, EMD Millipore, Darmstadt, GER) [48]. Cells were then treated with STI571 in the indicated concentrations for $48 \mathrm{hrs}$; for each treatment, $\mathrm{n}=6$ experiments were performed. Invasive tumour cells that have invaded through the ECM-covered pores along an FCS gradient to the bottom of the polycarbonate membrane and into the outer chamber and were trypsinised, spun on glass slides (Menzel, Braunschweig, GER) and counterstained with DAPI. Quantification of cells in 6 visual fields ( $\times 10$ objective lens) was done using Image J image analysis software (National Institutes of Health, Bethesda, USA).

\section{Statistics}

Differences in Abi1 IHC scores between invasion front/ tumour centre, tumours with different invasion patterns (expanding/low-grade budding, expanding/high-grade budding, infiltrating/low-grade budding and infiltrating/ high-grade budding) and tumours with and without lymphovascular infiltration were analysed using the KruskalWallis test followed by Dunn's multiple comparison posttest (SigmaPlot 12.0, Systat Software, Erkrath, Germany). For quantification of ECM degradation, mean degradation coefficients for 10-15 visual fields for each treatment/transfection were calculated. The differences between degradation coefficients after DMSO control/STI571 treatments and vector control/RNAi knockdown as well as between numbers of attached/invading cells in fibronectin adhesion/ ECMatrix cell invasion assay were tested for significance performing nonparametric ANOVA (Kruskal-Wallis) test using SigmaPlot 12.0 (Systat Software, Erkrath, Germany) with Dunn's multiple comparison post-test, where applicable. $P$ values $<0.05$ were regarded as statistically significant.

\section{Consent}

All cases that are reported in the manuscript were collected for histologic examination and diagnosis purpose and thoroughly anonymized for the use in this study. Informed consent was therefore not needed to be obtained. This study was approved by the instutional ethics committee of the University of Ulm (decision no. 162/13) and conforms to the current guidelines of the German Ethics Council.

\section{Additional file}

Additional file 1: Figure S1. Further analysis of Laminin5, E-cadherin and hnRNP K expression in CHD1 and HDC9 cells. A, western immunoblottig against Laminin5 (left), blots are shown after short (15 s) and long exposure
(10 min) and E-Cadherin (right). B, immunofluorescence stainings of hnRNP K/Phalloidin, Laminin5/Phalloidin and E-cadherin/Phalloidin. Scale bars as indicated. Figure S2. Verification of Abi1 RNAi knockdown in CHD1 CRC colorectal carcinoma cells. A, by immunofluorescence microscopy. B, by western immunoblotting (compared to pSuper vector control). Scale bars as indicated. Figure S3. Western immunoblotting of CHD1 cells after application of DMSO control as well as 5, 10 (A) and $50 \mu \mathrm{M}$ STI571 (B). A, with antibodies against Abi1 and $\beta$-Actin (loading control). B, with an antibody against cleaved caspase-3 (CC-3). While CC-3 is barely detectable after DMSO or after application of 5 or $10 \mu \mathrm{M}$ STI, there is strong immunoreactivity upon $50 \mu \mathrm{M}$ STI571. DMSO, dimethyl sulfoxide.

\section{Abbreviations}

Abi1: Abelson interactor 1; Abl: Abelson tyrosine kinase; Arp2/3: Actin-related protein 2/3; BRAF: V-Raf murine sarcoma viral oncogene homolog B1; CC-3: Cleaved caspase-3; CRC: Colorectal carcinoma; DMSO: Dimethyl sulfoxide; ECM: Extracellular matrix; F-actin: Filamentous actin; FCS: Fetal calf serum; HHR: Homeobox homology region; hnRNP K: Heterogeneous nuclear ribonucleoprotein K; HSPC300 (syn. BRICK1): SCARWAVE actin-nucleating complex subunit; KRAS: Kirsten rat sarcoma: MAPK: Mitogen-associated protein kinase; MMP: Matrix metalloproteinase; Nap: NCK-associated protein 1; NPF: Nucleation-promoting factor; PI3K: Phosphatidylinositol-3-kinase; SH3: Src homology domain 3; Sra (syn. PIR121): p53 inducible protein; UICC: Union international contre le cancer WAB: Wave-binding domain; (N-) WASPMAVE: (neural) Wiskott-Aldrich syndrome protein/ verprolin homologous.

\section{Competing interests}

The authors declare that they have no competing interests.

\section{Authors' contributions}

KS and KK carried out (immuno-) histologic analyses. KS and SB carried out in vitro analyses (cell culture, matrix degradation and cell invasion assays). KS carried out immunofluorescence microscopy. KS, JS and CP performed western immunoblotting. KS, JL and PM participated in the design of the study, and KS performed the statistical analyses. KS, JL, JS, VM and PM conceived of the study, and participated in its design and coordination and helped to draft the manuscript. All authors read and approved the final manuscript.

\section{Acknowledgements}

The authors would like to thank Claudia Schlosser, Verena Doucha, Anette Daniel and Jianqing Chen for expert technical assistance.

This study was supported by the young investigators academy of the German society of Pathology (KS).

\section{Author details}

'Bundeswehr Institute of Radiobiology, Neuherbergstr. 11, 80937 Munich, Germany. ${ }^{2}$ Institute of Pathology, University Hospital, Ulm, Germany. ${ }^{3}$ Clinic of Urology, University Hospital, Ulm, Germany. ${ }^{4}$ Institute of Pathology and Molecular Pathology, Bundeswehrkrankenhaus, UIm, Germany. ${ }^{5}$ Institute of Anatomy and Cell Biology, University of Ulm, Ulm, Germany.

Received: 4 February 2014 Accepted: 2 June 2014

Published: 9 June 2014

\section{References}

1. Brenner H, Bouvier AM, Foschi R, Hackl M, Larsen IK, Lemmens V, Mangone L, Francisci S: Progress in colorectal cancer survival in Europe from the late 1980s to the early 21st century: the EUROCARE study. Int I Cancer 2012, 131:1649-1658.

2. Jass JR, Love SB, Northover JM: A new prognostic classification of rectal cancer. Lancet 1987, 1:1303-1306.

3. Ueno H, Murphy J, Jass JR, Mochizuki H, Talbot IC: Tumour 'budding' as an index to estimate the potential of aggressiveness in rectal cancer. Histopathology 2002, 40:127-132.

4. Linder S, Wiesner C, Himmel M: Degrading devices: invadosomes in proteolytic cell invasion. Annu Rev Cell Dev Biol 2011, 27:185-211.

5. Ridley AJ: Life at the leading edge. Cell 2011, 145:1012-1022. 
6. Le Clainche C, Carlier MF: Regulation of actin assembly associated with protrusion and adhesion in cell migration. Physiol Rev 2008, 88:489-513.

7. Courtney KD, Grove M, Vandongen H, Vandongen A, LaMantia AS, Pendergast AM: Localization and phosphorylation of Abl-interactor proteins, $\mathrm{Abi}-1$ and $\mathrm{Abi}-2$, in the developing nervous system. Mol Cell Neurosci 2000, 16:244-257.

8. Proepper C, Johannsen S, Liebau S, Dahl J, Vaida B, Bockmann J, Kreutz MR Gundelfinger ED, Boeckers TM: Abelson interacting protein 1 (Abi-1) is essential for dendrite morphogenesis and synapse formation. EMBO 2007, 26:1397-1409.

9. Proepper C, Steinestel K, Schmeisser MJ, Heinrich J, Steinestel J, Bockmann J, Liebau S, Boeckers TM: Heterogeneous nuclear ribonucleoprotein k interacts with Abi-1 at postsynaptic sites and modulates dendritic spine morphology. PLoS One 2011, 6:e27045.

10. Ito H, Morishita R, Shinoda T, Iwamoto I, Sudo K, Okamoto K, Nagata K. Dysbindin-1, WAVE2 and Abi-1 form a complex that regulates dendritic spine formation. Mol Psychiatry 2010, 15:976-986.

11. Innocenti M, Gerboth S, Rottner K, Lai FP, Hertzog M, Stradal TE, Frittoli E, Didry D, Polo S, Disanza A, Benesch S, Di Fiore PP, Carlier MF, Scita G: Abi1 regulates the activity of N-WASP and WAVE in distinct actin-based processes. Nat Cell Biol 2005, 7:969-976.

12. Takenawa T, Suetsugu S: The WASP-WAVE protein network: connecting the membrane to the cytoskeleton. Nat Rev Mol Cell Biol 2007, 8:37-48.

13. Dubielecka PM, Ladwein Kl, Xiong X, Migeotte I, Chorzalska A, Anderson KV, Sawicki JA, Rottner K, Stradal TE, Kotula L: Essential role for Abi1 in embryonic survival and WAVE2 complex integrity. Proc Natl Acad Sci U S A 2011, 108:7022-7027.

14. Dubielecka PM, Machida K, Xiong X, Hossain S, Ogiue-lkeda M, Carrera AC, Mayer BJ, Kotula L: Abi1/Hssh3bp1 pY213 links Abl kinase signaling to p85 regulatory subunit of $\mathrm{PI}-3$ kinase in regulation of macropinocytosis in LNCaP cells. FEBS Lett 2010, 584:3279-3286.

15. Kotula L: Abi1, a critical molecule coordinating actin cytoskeleton reorganization with PI-3 kinase and growth signaling. FEBS Lett 2012, 586:2790-2794.

16. Sun X, Li C, Zhuang C, Gilmore WC, Cobos E, Tao Y, Dai Z: Abl interactor 1 regulates Src-Id1-matrix metalloproteinase 9 axis and is required for invadopodia formation, extracellular matrix degradation and tumor growth of human breast cancer cells. Carcinogenesis 2009, 30:2109-2116.

17. Hossain S, Dubielecka PM, Sikorski AF, Birge RB, Kotula L: Crk and ABI1: binary molecular switches that regulate abl tyrosine kinase and signaling to the cytoskeleton. Genes Cancer 2012, 3:402-413.

18. Bellone G, Ferrero D, Carbone A, de Quadros MR, Gramigni C, Prati A Davidson W, Mioli P, Dughera L, Emanuelli G, Rodeck U: Inhibition of cell survival and invasive potential of colorectal carcinoma cells by the tyrosine kinase inhibitor STI571. Cancer Biol Ther 2004, 3:385-392.

19. Smith-Pearson PS, Greuber EK, Yogalingam G, Pendergast AM: Abl kinases are required for invadopodia formation and chemokine-induced invasion. J Biol Chem 2010, 285:40201-40211.

20. Attoub S, Rivat C, Rodrigues S, van Bocxlaer S, Bedin M, Bruyneel E, Louvet C, Kornprobst M, Andre T, Mareel M, Mester J, Gespach C: The c-kit tyrosine kinase inhibitor STI571 for colorectal cancer therapy. Cancer Res 2002, 62:4879-4883.

21. Popow-Wozniak A, Wozniakowska A, Kaczmarek L, Malicka-Blaszkiewicz M, Nowak D: Apoptotic effect of imatinib on human colon adenocarcinoma cells: influence on actin cytoskeleton organization and cell migration. Eur J Pharmacol 2011, 667:66-73.

22. Steinestel K, Bruderlein S, Steinestel J, Markl B, Schwerer MJ, Arndt A, Kraft K, Propper C, Moller P: Expression of Abelson interactor 1 (Abi1) correlates with inflammation, KRAS mutation and adenomatous change during colonic carcinogenesis. PLoS One 2012, 7:e40671.

23. Carpenter B, McKay M, Dundas SR, Lawrie LC, Telfer C, Murray GI: Heterogeneous nuclear ribonucleoprotein $\mathrm{K}$ is over expressed, aberrantly localised and is associated with poor prognosis in colorectal cancer. Br J Cancer 2006, 95:921-927.

24. Klimek-Tomczak K, Mikula M, Dzwonek A, Paziewska A, Karczmarski J, Hennig E, Bujnicki JM, Bragoszewski P, Denisenko O, Bomsztyk K, Ostrowski J: Editing of hnRNP K protein mRNA in colorectal adenocarcinoma and surrounding mucosa. Br J Cancer 2006, 94:586-592.

25. Iwaya K, Oikawa K, Semba S, Tsuchiya B, Mukai Y, Otsubo T, Nagao T, Izumi M, Kuroda M, Domoto H, Mukai K: Correlation between liver metastasis of the colocalization of actin-related protein 2 and 3 complex and WAVE2 in colorectal carcinoma. Cancer Sci 2007, 98:992-999.
26. Yanagawa R, Furukawa Y, Tsunoda T, Kitahara O, Kameyama M, Murata K, Ishikawa O, Nakamura Y: Genome-wide screening of genes showing altered expression in liver metastases of human colorectal cancers by cDNA microarray. Neoplasia (New York, NY) 2001, 3:395.

27. Giannelli G, Falk-Marzillier J, Schiraldi O, Stetler-Stevenson WG, Quaranta V: Induction of cell migration by matrix metalloprotease-2 cleavage of laminin-5. Science 1997, 277:225-228.

28. Stradal T, Courtney KD, Rottner K, Hahne P, Small JV, Pendergast AM: The $\mathrm{Abl}$ interactor proteins localize to sites of actin polymerization at the tips of lamellipodia and filopodia. Curr Bio/ 2001, 11:891-895.

29. Chen H, Wu X, Pan ZK, Huang S: Integrity of SOS1/EPS8/ABI1 tri-complex determines ovarian cancer metastasis. Cancer Res 2010, 70:9979-9990.

30. Wang C, Tran-Thanh D, Moreno JC, Cawthorn TR, Jacks LM, Wang DY, McCready DR, Done SJ: Expression of Abl interactor 1 and its prognostic significance in breast cancer: a tissue-array-based investigation. Breast Cancer Res Treat 2011, 129:373-386.

31. The GeneSapiens database. [http://www.genesapiens.org]

32. The human protein atlas. [http://www.proteinatlas.org]

33. Uhlen M, Oksvold P, Fagerberg L, Lundberg E, Jonasson K, Forsberg M, Zwahlen M, Kampf C, Wester K, Hober S, Wernerus H, Björling L, Ponten F: Towards a knowledge-based Human Protein Atlas. Nat Biotechnol 2010, 28:1248-1250

34. Uhlen $\mathrm{M}$, Bjorling $\mathrm{E}$, Agaton $\mathrm{C}$, Szigyarto CA, Amini B, Andersen $\mathrm{E}$, Andersson AC, Angelidou P, Asplund A, Asplund C, Berglund L, Bergström K, Brumer $\mathrm{H}$, Cerjan D, Ekström M, Elobeid A, Eriksson C, Fagerberg L, Falk R, Fall J, Forsberg M, Björklund MG, Gumbel K, Halimi A, Hallin I, Hamsten C, Hansson M, Hedhammar M, Hercules G, Kampf C, et al: A human protein atlas for normal and cancer tissues based on antibody proteomics. $\mathrm{Mol}$ Cell Proteomics 2005, 4:1920-1932.

35. Betge J, Pollheimer MJ, Lindtner RA, Kornprat $P$, Schlemmer A, Rehak $P$, Vieth M, Hoefler G, Langner C: Intramural and extramural vascular invasion in colorectal cancer: prognostic significance and quality of pathology reporting. Cancer 2012, 118:628-638.

36. Remy L, Trespeuch C, Bachy S, Scoazec JY, Rousselle P: Matrilysin 1 influences colon carcinoma cell migration by cleavage of the laminin- 5 beta3 chain. Cancer Res 2006, 66:11228-11237.

37. Makrodouli E, Oikonomou E, Koc M, Andera L, Sasazuki T, Shirasawa S, Pintzas A: BRAF and RAS oncogenes regulate Rho GTPase pathways to mediate migration and invasion properties in human colon cancer cells: a comparative study. Mol Cancer 2011, 10:118

38. Lin Y-N, Izbicki JR, König A, Habermann JK, Blechner C, Lange T, Schumacher U, Windhorst S: Expression of DIAPH1 is up-regulated in colorectal cancer and its down-regulation strongly reduces the metastatic capacity of colon carcinoma cells. Int J Cancer 2014, 134:1571-1582.

39. Giannelli G, Antonaci S: Biological and clinical relevance of Laminin-5 in cancer. Clin Exp Metastasis 2000, 18:439-443.

40. Kim YW, Ko YT, Kim NK, Chung HC, Min BS, Lee KY, Park JP, Kim H: A comparative study of protein expression in primary colorectal cancer and synchronous hepatic metastases: the significance of matrix metalloproteinase-1 expression as a predictor of liver metastasis. Scand J Gastroenterol 2010, 45:217-225.

41. Murray GI, Duncan ME, O'Neil P, Melvin WT, Fothergill JE: Matrix metalloproteinase-1 is associated with poor prognosis in colorectal cancer. Nat Med 1996, 2:461-462.

42. Kilpinen S, Autio R, Ojala K, Iljin K, Bucher E, Sara H, Pisto T, Saarela M, Skotheim Rl, Bjorkman M, Mpindi JP, Haapa-Paananen S, Vainio P, Edgren $\mathrm{H}$, Wolf M, Astola J, Nees M, Hautaniemi S, Kallioniemi O: Systematic bioinformatic analysis of expression levels of 17,330 human genes across 9,783 samples from 175 types of healthy and pathological tissues. Genome Biol 2008, 9:R139.

43. Moller P, Koretz K, Leithauser F, Bruderlein S, Henne C, Quentmeier A, Krammer PH: Expression of APO-1 (CD95), a member of the NGF/TNF receptor superfamily, in normal and neoplastic colon epithelium. Int J Cancer 1994, 57:371-377.

44. Liebau S, Steinestel J, Linta L, Kleger A, Storch A, Schoen M, Steinestel K, Proepper C, Bockmann J, Schmeisser MJ, Boeckers TM: An SK3 channel/ nWASP/Abi-1 complex is involved in early neurogenesis. PLoS One 2011 6:e18148.

45. Collins TJ: ImageJ for microscopy. Biotechniques 2007, 43:25-30

46. Artym W, Yamada KM, Mueller SC: ECM degradation assays for analyzing local cell invasion. Methods Mol Biol 2009, 522:211-219. 
47. Chu CY, Rana TM: Potent RNAi by short RNA triggers. RNA 2008, 14:1714-1719.

48. Terranova VP, Hujanen ES, Loeb DM, Martin GR, Thornburg L, Glushko V: Use of a reconstituted basement membrane to measure cell invasiveness and select for highly invasive tumor cells. Proc Natl Acad Sci U S A 1986, 83:465-469.

doi:10.1186/1476-4598-13-145

Cite this article as: Steinestel et al:: Expression and Y435-phosphorylation of Abelson interactor 1 (Abi1) promotes tumour cell adhesion, extracellular matrix degradation and invasion by colorectal carcinoma cells. Molecular Cancer 2014 13:145.

\section{Submit your next manuscript to BioMed Central and take full advantage of:}

- Convenient online submission

- Thorough peer review

- No space constraints or color figure charges

- Immediate publication on acceptance

- Inclusion in PubMed, CAS, Scopus and Google Scholar

- Research which is freely available for redistribution 